\title{
Evolution of Orchestration Towards 5G
}

\author{
Kasra Kassai, Saptarshi Ghosh, and Tasos Dagiuklas \\ London South Bank University, London SE1 0AA, UK \\ Email: kasra.kassai@\{1sbu/city\}.ac.uk; ghoshs4@1sbu.ac.uk; tdagiukas@1sbu.ac.uk
}

\begin{abstract}
Service orchestration is an essential activity in 5G networks. It performs optimal resource allocation and provisions services in an effective sequence based on demands across a collection of physical or virtual network functions (P/VNF). This paper summarizes several orchestration environments and components along with their evolution towards 5G. A brief operational comparison of platforms such as Open Source Management and Orchestration (OSM MANO), Open Platform for NFV (OPNFV) and Open Network Automation Platform (ONAP) have been presented, along with different deployment models and architectural alternatives.
\end{abstract}

Index Terms - Network Architecture; Network Slicing; Orchestration; Network Softwarisation; 5G; NFV; MANO; ETSI; SDN; MEC;

\section{INTRODUCTION}

In recent years, the rapid development of many innovative technological advancements in networks has taken place. Eventually, data traffic, connectivity demands and is skyrocketing. The evolution of the 5th Generation (5G) expects to have more than 10 to 100 times more connected devices. The total data traffic for all devices carried by the only $5 \mathrm{G}$ is anticipated to increase by more than 34 times during 2019 until the end of 2024 reaching the monthly data traffic of 34 Exabytes; whereas, all data traffic worldwide carried out by $2 \mathrm{G} / 3 \mathrm{G} / 4 \mathrm{G}$ networks during this forecast period is expected to be only about 3 times more than the total traffic today [1].

The realisation of the $5 \mathrm{G}$ infrastructure relies on three key technological areas: enhanced Mobile Broadband (eMBB), Ultra- reliable and Low Latency Communication (uRLLC), and Massive Machine Type Communication (mMTC). Additionally, the need of 5G networks necessitates support Internet of Things (IoT) [2] in the context of Heterogeneous Network (HetNet) [3], Cloud Radio Access Networks (cRAN), and Machine to Machine (M2M) communications [4].

The $5 \mathrm{G}$ architecture is designed to realise scalable and flexible network deployment and operations with major differences than the previous generation. One of the main challenges on the way to achieve the desired flexibility to address requirements for a wide variety of scenarios and applications characteristics and specifications. Network Softwarisation is a key design concept that serves to tackle this problem [5]. Software-Defined Network (SDN), Network Function Virtualization (NFV) and Multi-Access Edge Computing (MEC) are becoming a prominent part of high- level next-generation network architecture. SDN decouples the control and data plane that enhances programmability and offers centralised management, also it reduces the CapEx and OpEx [6]. In NFV the hardwarebased functionalities are migrated to virtual manageable software units. The idea is to virtualize and manage resources, which are shared but are isolated and allocated virtually by the controller. Multi-tenancy is one of the other desirable features of new networks. Network Slicing is an attractive solution and a trending topic of research in academia.

Network Slicing can be significantly supported by NFV and SDN, where network functions are virtualised and can be managed and controlled using cloud and implemented using a logical central controller. This helps to create new solutions and to provide value to the industry and besides reduction of CAPEX and OPEX [6]. For each slice as per specific application, storage and computation entities and VNFs must be allocated and provisioned. For instance, a slice allocated to serve eMBB requires a wider bandwidth than a slice assigned for a real-time uRRLC application, which is more delay-sensitive.

A well-defined management and orchestration architecture is necessary to administer network resources. The most notable Management and Orchestration (MANO) activity is being conducted by ETSI NFV MANO, a standard organization body. ETSI MANO framework is a 3-tier framework: 1- Architecture 2MANO and 3- Network management. This framework is further explained in more details in the next section.

For telecommunication operators a reliable and dynamic MANO framework is vital. They need to support services powered by NFV environment. An orchestrator is responsible for the placement of NFVs and optimises the uses of NFV Infrastructure. The schema and mapping of resources to applications enables orchestrators for faulttolerance, availability and ability for recovery.

The paper is structured as follows. In section 2, we focus on recently emerged architecture and how this can leverage computing, networking and storage resources to meet user requirements. In section 3 , we present the important role of network slicing in the architecture. In Section 4, the need for orchestration entity is explained. 


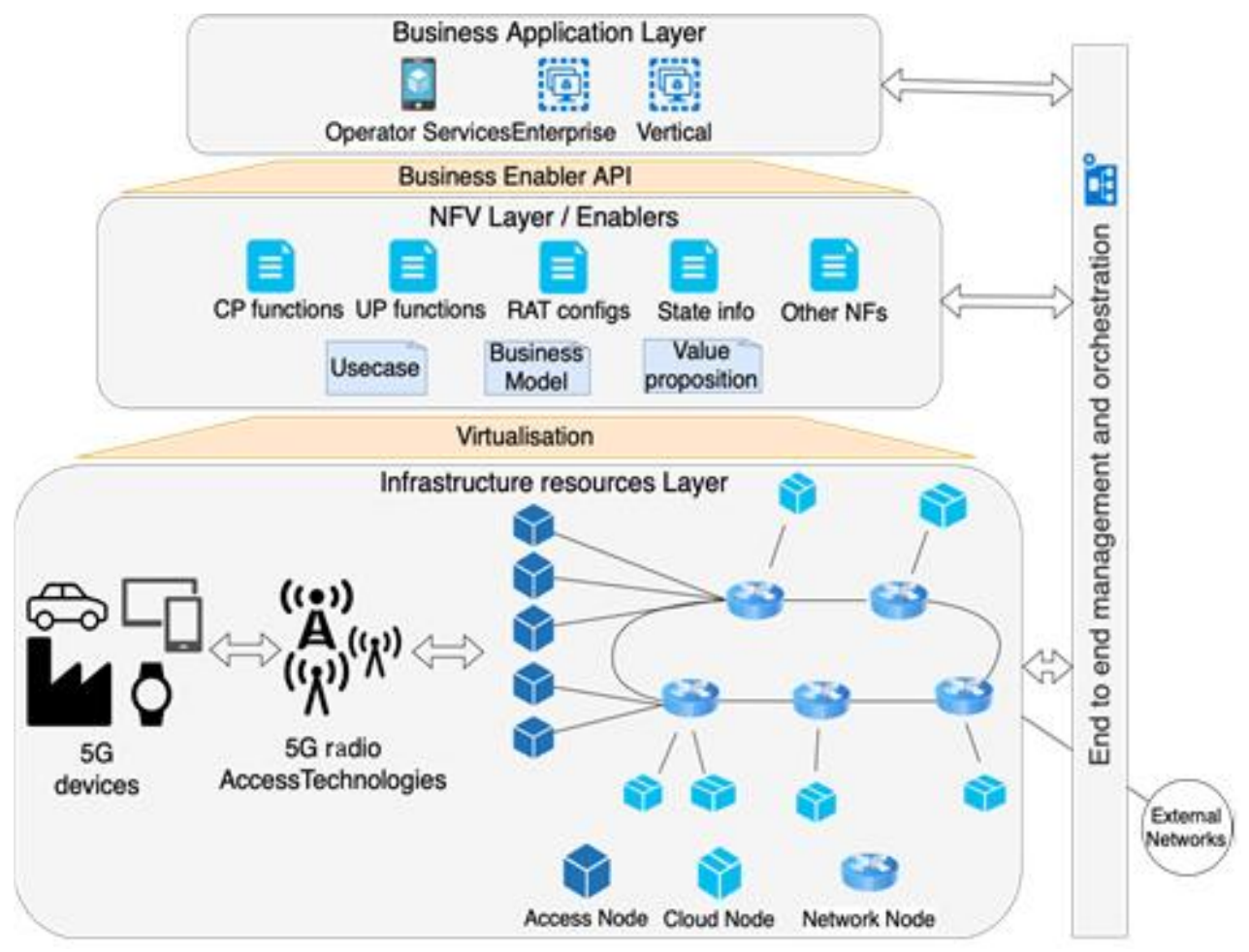

Fig. 1. Network Softwarisation Architecture

\section{SOFTWARISATION IN 5G}

Recent network architectures have evolved to cater to NFV [7], Edge Cloud Computing (i.e. Fog [8] and MEC [9][10]), SDN and new arising technologies and incorporated in modern network designs. We are facing a convergence of computing and communication resources that results in an end-to-end virtualised service delivery.

For supporting dynamic service chaining in the $5 \mathrm{G}$ architecture, an on-demand, scalable and flexible design is necessary. The recent architectures with NFV and SDN components included are not enough to serve desired flexibility and scalability. Fig. 2 illustrates a Network Softwarisation architecture. The architecture clearly depicts the separation of software and the physical infrastructure. This architecture comprises the following:

\section{A. Infrastructure Resources Layer}

Physical resources including cloud and access nodes, smartphones and customer premises equipment (CPE). Through APIs, this layer as shown in Fig. 1 exposes services to top layers (Enablers layer and business

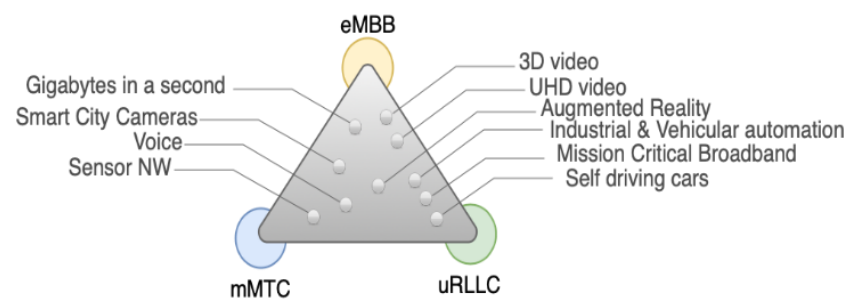

Fig. 2. 5G Use-cases application layer) and to management and orchestration entity.

\section{B. NFV Layer}

The concept of NFV is around since October 2012. In this layer packages of Virtual Network Functions (NFVs) are deployed virtually. A deployment to service a use-case can be made up of several VNFs that are chained to each other. This means that some VNFs may be provisioned first to satisfy the requirements of the other

NFVs to be deployed afterwards.

\section{Business Application Layer}

Service management must be able to handle the challenges caused by NFV service chainings, such as maintaining multiple network service chains and service chain dependency management. [11]. Mobile Network

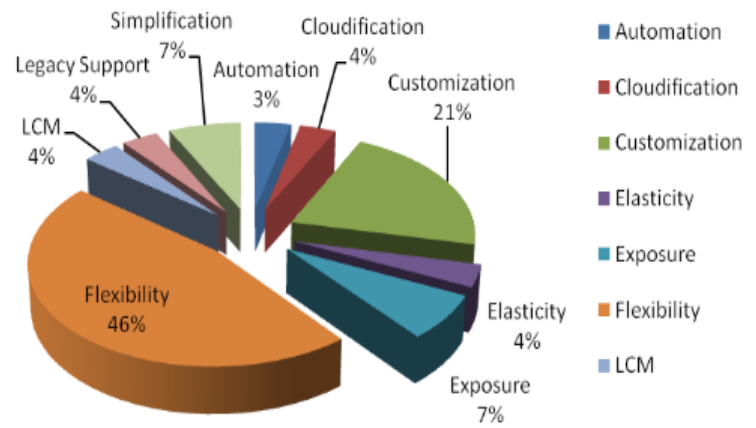

Fig. 3. Network Slicing Requirements [15] 
Operator (MNO) subscribers, enterprise and other industry verticals can utilise services through APIs exposed from NFV layer

\section{The Orchestrator}

The only entity aware of all three layers is orchestrator, thus utilizes management tools to automate service deployment, guarantee SLA, QoS and ensures maintainability of the infrastructure. In such a multi-layer architecture, maintainability, management and reliability are served by management and orchestration (MANO) component. Mobile Network Operator (MNO) subscribers, enterprise and other industry verticals can utilise services through APIs exposed from NFV layer.

\section{NETWORK SLICING}

The 5GPPP defines network slice as "A composition of adequately configured network functions, network applications, and the underlying cloud infrastructure (physical, virtual or even emulated resources, RAN resources etc.), that are bundled together to meet the requirements of a specific use case, e.g., bandwidth, latency, processing, and resiliency, coupled with a business purpose." Network-as-a-Service paradigm, where network resources are on-demand is highly reliant on SDN and NFV. Automatically managing partitioned networks (slices), can also lead to a straightforward NFV deployment.

Network slicing can facilitate building a dedicated and isolated portion of virtualized resources of the network, logically to support multiple user groups or applications with different specifications and accommodate multitenancy. For instance, eMBB slice, IoT slice and mMTC slice. Telemedicine will be allocated on a network slice, which is different from the slice serving video streaming services.

NGMN considers network slicing a concept which can realise a network service when hosted by several virtual and non-virtual resources required for that service. Like NFV, each slice must be created and instantiated by a controller. The instantiated object spans overall network layers to encapsulate a full set of virtual and nonvirtual computing and networking resources to meet an application scenario. A collection of VNFs of a slice in the business enabler layer. A collection of Radio Access Technologies and their configurations are also packaged and allocated to the instance. In the Business Application layer, the slice comprises either current Software as a Service cloud nodes or API-enabled proprietary applications.

Adding more importance and vitality to service management is critical, the service management for slices must have a sight on all network layers due to the end-toend nature of network slicing. In centralised network management and orchestration, the orchestrator is in communication with micro-services(slices) and with the VNF. Management requirements for realisation of network slicing are elasticity, cloudification and exposure of APIs which are currently is missing (Fig. 3), flexibility $(46 \%)$ and customisation $(21 \%)$ are the two most highlighted.

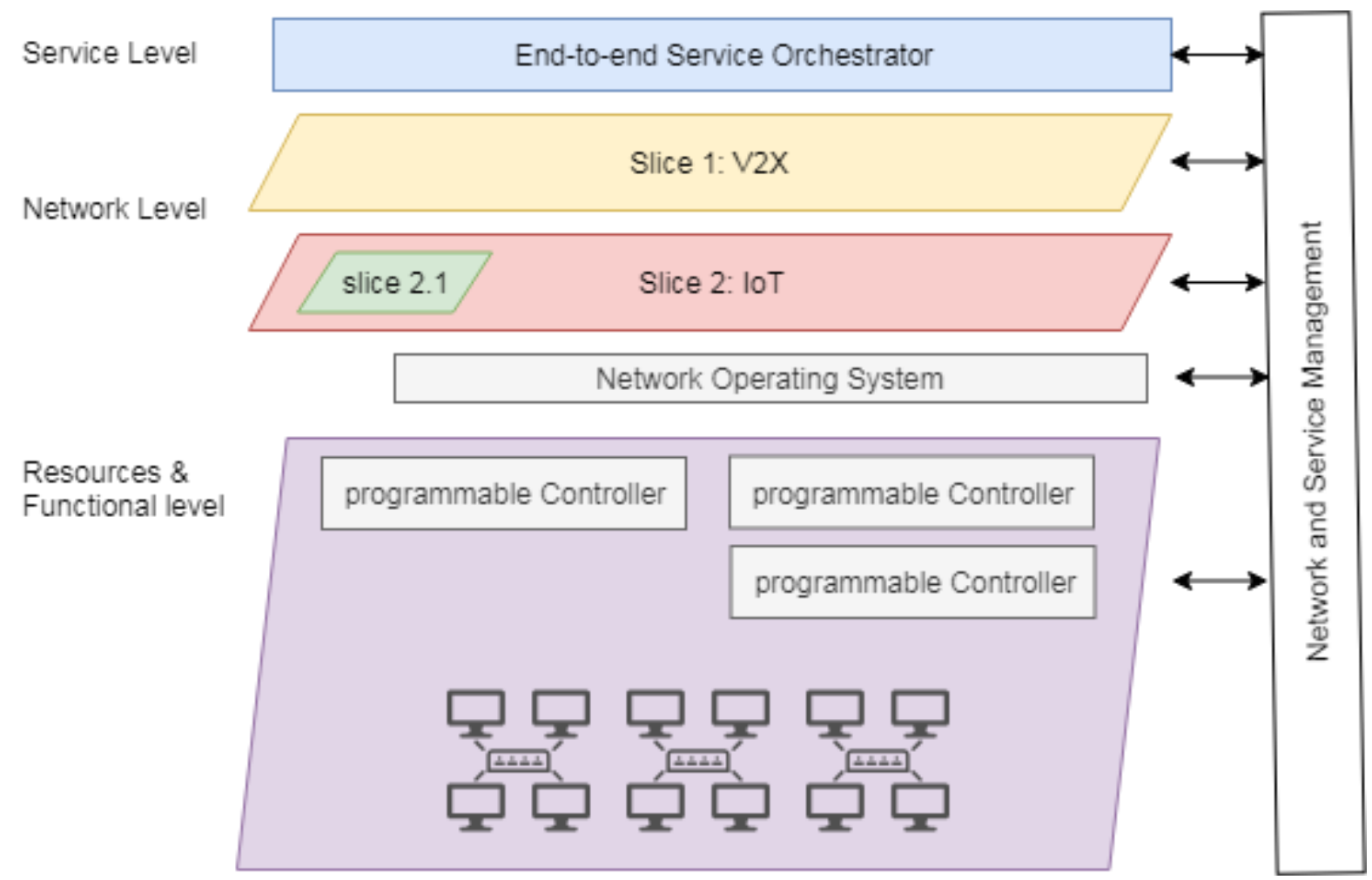

Fig. 4. Network Slicing View 


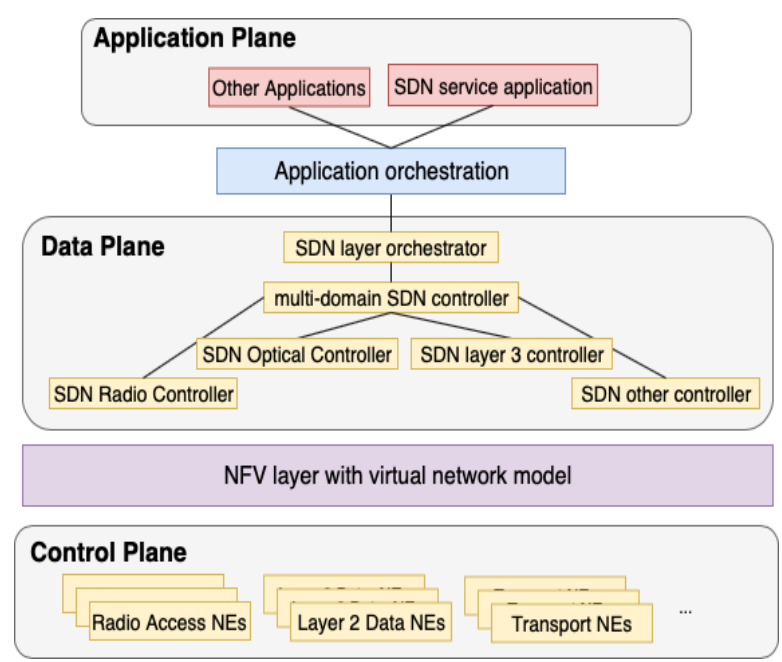

Fig. 5. components in different layers

\section{THE NEED FOR ORCHESTRATION}

A very good definition for orchestration by 5GPPP is a component that "describes the automated arrangement, coordination, and management of complex systems, middleware and services". Network orchestration is responsible to carry out the following:

\section{A. Deployment}

Sharing telecom infrastructure has become an attractive option, and the saved money can be spent on shipping more network services or delivering better QoS.

More in details, the deployment and management of VNFs is a crucial task.

\section{B. Operation}

The control and operation of resources can be reached through orchestration.

\section{Life Cycle Management}

Initiates majority of requests in a sequence to fully deploy a service. (Fig. 4). The orchestrated resources will result in specific business models' requirements to be met. The full potential of what network can offer will be unlocked. For network service instantiation BSS/OSS component will submit a request for deploying a new service to Service Lifecycle Manager (SLM). Then SLM requests service and functional descriptors from the catalogue. Catalogue responses with the requested descriptors. SLM now send a message to the resource manager requesting resource information from it.

Once response received from the resource manager, SLM requests for placement calculations from placement manager, so now can request VNF manager which is outside the orchestrator to allocate resources and complete deployment based on configurations of descriptors and on specified locations. Monitoring manager now gets triggered and OSS/BSS gets notified that the service is deployed. [12]

\section{STANDARDS FOR ORCHESTRATION}

The goal for Standard Setting Organizations (SSOs) is to ensure that Quality of Service (QoS), throughput and performance demands are satisfied, and different domains can inter-operate and build on the same common agreements and as result reduce CAPEX and OPEX. In such a standard framework, flexibility is a key feature to satisfy diverse user requirements in the $5 \mathrm{G}$ era. The diversity of requirements is both in the traffic pattern and resource requirements. Additionally, they are dynamically changing over time and the change is not identical for different user groups.

Knowing that $5 \mathrm{G}$ will be rolled out completely after 2020, each industry is trying to get its own bit. The benefit is very important to match the specifications of any design or product with the SSO. 3GPP comprises seven organizational partners and publishes technical specifications and standards. ITU coordinates worldwide

technical communication standards and IETF is for producing Internet standards and guidelines. IETF has also focused on 5G. NGMN $5 \mathrm{G}$ initiative was a project by NGMN 5G team. They published the 1st whitepaper on 5G. According to NGMN network cloud orchestration (Fig. 5) is a necessity to deliver network availability and reliability KPIs [13]

ETSI NFV architecture is described by ETSI-NFV and ETSI- MANO is the standard framework for management and orchestration architectures in 5G. The importance of compliance with standards is even more when it comes to later interoperability of virtual functions and end-to-end virtual network dedication. [14] ETSI NFV MANO consists of data models and standards in following: [15]

- NFV Orchestration: It manages the lifecycle of Network Service. It is also called NFVO.

- VNF manager: It manages the lifecycle of VNF instances. "Fault, Configuration, Accounting, Performance and Security" of VNF instances.

- Virtualised Infrastructure Manager: It handles VNFI resources.

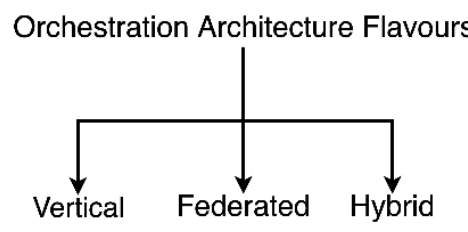

Fig. 6. Types of Orchestration Architecture

In this section, based on the complexity and service characteristics of the network, the orchestration architecture can be of three types: (Fig. 6) 
- Vertical or hierarchical orchestration is a top-down approach that starts from a business level and goes all the way to resource instances. It provides on-demand services like synchronisation and delegation across other layers. This makes the architecture scalable and elastic.

- Federated orchestration is catered when the end-toend visibility over a multi-domain network cannot be achieved. Each domain orchestrates with a dedicated controller or orchestrator and performs harmony between them. Unlike vertical orchestration, here the interfaces are horizontal.

- Hybrid orchestration clubs the two types mentioned above. To achieve end-to-end service delivery, both vertical and horizontal orchestrations are partially deployed to control the entire network. Typically, the above types are implemented on top of the lower and upper layers respectively. Therefore, it blends the autonomy from the vertical and the federation capabilities offered by the horizontal orchestration.

In this section, the different configurations of administrative domains and its deployment model for network slices are discussed. (Fig. 7)

- Intra-domain deployment is done on small-scale and less-complex networks that comprise of one or more sub-administrative domains. In such a setup, a single policy acts upon the entire domain. therefore, a single orchestrator adequate the implementation. To deploy multiple policies over the sub-domains slices can be created and designated to more orchestrators.

- Inter-domain deployment is suitable for a network where two administrative domains are provisioned. Network slices coordinate across the domains by the cooperation and the synchronisation of the respected orchestrators. This includes Service-Level Agreements (SLA) between them. (Fig. 7)

- Multi-domain refers to the most complex setup where more than two administrative domains take part. Several resources and policies must be synchronised to cater to a specific service. coordination across domain-specific orchestrators

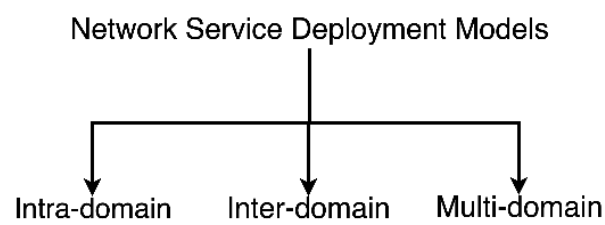

Fig. 7. Network Service Deployment Models

plays a key role in such environments, through SLA on different policies designated on the slices.

Based on the service characteristics, each domain configurations mentioned above may accumulate service

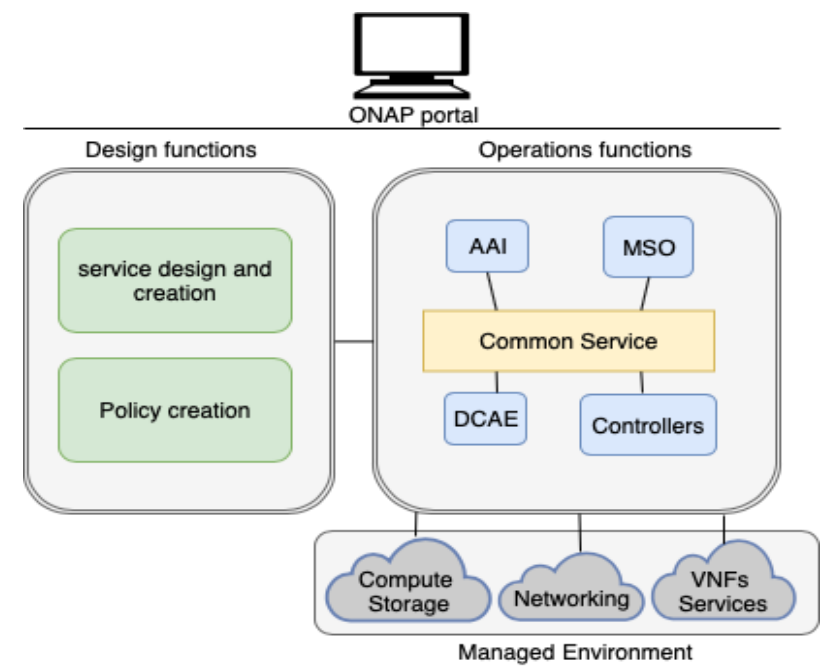

Fig. 8. ONAP architecture in a glance

prover and network service provider or just network service providers.

\section{A. OSM MANO}

Open Source MANO (OSM) is an ETSI-hosted initiative for the development of open-source NFV Management and Orchestration software stacks. The architecture of OSM consists of two major components: run-time scope and design-time scope.

OSM MANO leverages three orchestration components to build an all-in-one MANO framework.

\section{- Telefonica's OpenMANO project}

\section{- Canonical's Juju generic VNF Manager}

\section{- RIFT.io's orchestrator}

OSM did not start from the scratch it was initially compiling existing parts into the unified model.

OpenMANO was the seed resource orchestrator, Riftware from Rift.io as seed service orchestrator and Juju as an external reference for VNF configuration and management. Juju VNF manager can deploy OpenBaton VNF packages and Juju charms, A Juju charm contains all the instructions necessary for deploying and configuring model. OpenMANO was the seed resource orchestrator, Riftware from Rift.io as seed service orchestrator and Juju as an external reference for VNF configuration and management. Juju VNF manager can deploy OpenBaton VNF packages and Juju charms. A Juju charm contains all the instructions necessary for deploying and configuring application units.

They are available on the Charm Store and can scale up and down. The simplest application can be a bundled charm using a Juju client to deliver infrastructure collection for an application scenario. 
Rift's role is in orchestrator component, by creating and instantiating VNFDs and NSDs for resiliency and scale it can provision many computer instances across data centres in a single environment.

OSM released its first release called release "ZERO" as in May 2016. The primary features were a simplified way of onboarding VNFs, utilising human readable VNF and network service (NS) descriptors. Multi VIM support, OpenStack and OpenVIM only at that time. Configuration with SDN and a web interface.

In release one, Multi-SDN, and plugin model introduces where OSM MANO can communicate with other different clouds and controllers. Network Service (NS) scaling was introduced and completed in release three. In the third release, interoperability on VIM improved and depreciated APIs by OpenStack has been removed from OSM. In release four, cloud-native install and more monitoring tools are integrated. And in release "FIVE" 5G use-cases, micro-services and more importantly full support of awaited 5G technology, network slicing is provided.

\section{B. ONAP AT\&T ECOMP AND LINUX FOUNDATION}

Open Network Automation Platform (ONAP) (Fig. 8) manages service creation of VNFs, deployment, storage and network resources. They don't differentiate resources by type and unifies them to schedule and implement tasks based on policies.

Design-time environment: The main role of the design time framework is to describe the design functions in meta data descriptions through the ONAP Portal. Basically, this component defines recipes for instantiating, monitoring and managing VNFs and services. It is also responsible for the distribution of these specific design rules into the execution time component. Execution-time environment: The execution time framework contains meta-data driven modules enabling VNF configuration and instantiation and delivers a real-time view of available resources and services. Unified design framework supporting TOSCA and YANG. Follows ETSI standard for interface and features and building blocks.

\section{OPNFV}

The Open Platform for NFV (OPNFV) project was introduced in September 2014 as an outgrowth of the ETSI NFV Industry Specification Group (ETSI NFV ISG). [16]

Establishment of OPNFV resulted from the realization that an open reference platform was needed to validate key NFV concepts, leverage the growing open source community, and accelerate development and ultimately adoption of NFV products and services.

OPNFV is now more of a Virtual Infrastructure Manager (VIM) that can be plugged into OSM MANO service provisioning. OpenStack, VMware and OpenDaylight are other VIMs.

Table of comparison between different opensource tools and packages here is showing how simple are the architecture based on number of components:

Rifttio.com contributes to NSO and UI and system

TABLE I: COMPARISON TABLE

\begin{tabular}{|c|c|c|c|c|}
\hline & OSM MANO & $\begin{array}{c}\text { OpenStack } \\
\text { Heat }\end{array}$ & Juju & Riftio \\
\hline Focus & $\begin{array}{c}\text { Full } \\
\text { framework }\end{array}$ & VIM & VNFM & NSO \\
\hline $\begin{array}{c}\text { Compliance } \\
\text { with ETSI }\end{array}$ & High & Low & High & High \\
\hline Simplicity & Low & High & Low & High \\
\hline Open Source & Yes & Yes & Yes & Yes \\
\hline
\end{tabular}

visibility layer of OSM MANO.

\section{CONCLUSION}

In this paper several orchestration environments and components for $5 \mathrm{G}$ networks have been summarised. The brief overview of the evolution towards $5 \mathrm{G}$ has been discussed. Various orchestration architectures as well as different deployment models have been presented. This paper serves the reader a premier of several orchestration techniques and implementation strategies. Ultimately, it illustrates network life cycle management and network slicing and at a glance. The future works can focus on open issues, such as: cross-domain orchestration, VNF placement for reliable service function chains and VNF management to manage the lifecycle of VNF instances.

\section{VII.ACKNOWLEDGMENT}

This work was supported in part by "Self-Organization toward reduced cost and energy per bit for future Emerging radio Technologies"' with contract number 734545 . The project has received research funding from the H2020MSCA-RISE-2016 European Framework Program. 


\section{REFERENCES}

[1] Ericsson, "Ericsson Mobility Visualizer: Interactive mobility data app." [Online]. available: https://www.ericsson.com/en/mobility-report/mobilityvisualizer. [Accessed: 12-Jan-2019].

[2] N. Shahid and S. Aneja, "Internet of Things: Vision, application areas and research challenges," Proc. Int. Conf. IoT Soc. Mobile, Anal. Cloud, I-SMAC 2017, vol. 10, no. 7, pp. 583-587, 2017.

[3] T. Qiu, N. Chen, K. Li, M. Atiquzzaman, and W. Zhao, "How can heterogeneous internet of things build our future: A survey," IEEE Commun. Surv. Tutorials, vol. 20, no. 3, pp. 2011-2027, 2018.

[4] W. G. 5GPPP Architecture, "5GPPP Architecture Working Group View on 5G Architecture," 2017.

[5] 5G PPP SN Working Group, "Vision on Software Networks and 5G," 5G-PPP Initiat., no. January, pp. 1- 38, 2017.

[6] J. Erfanian et al., "Network Functions Virtualisation White Paper on NFV priorities for 5G," ETSI White Pap., no. 1, pp. 1-15, 2017.

[7] B. Yi, X. Wang, K. Li, S. k. Das, and M. Huang, "A comprehensive survey of Network Function Virtualization," Comput. Networks, vol. 133, pp. 212-262, 2018.

[8] M. Wang, J. Chen, E. Aryafar, and M. Chiang, "A Survey of Client-Controlled HetNets for 5G," IEEE Access, vol. 5, pp. 2842-2854, 2017.

[9] N. Abbas, Y. Zhang, A. Taherkordi, and T. Skeie, "Mobile Edge Computing: A Survey," IEEE Internet Things J., vol. 5, no. 1, pp. 450-465, 2018.

[10] E. E. Ugwuanyi, S. Ghosh, M. Iqbal, and T. Dagiuklas, "Reliable Resource Provisioning Using Bankers' Deadlock Avoidance Algorithm in MEC for Industrial IoT," IEEE Access, vol. 6, pp. 43327-43335, 2018.

[11] H. Jeon and B. Lee, "Network service chaining challenges for VNF outsourcing in network function virtualization," in International Conference on ICT Convergence 2015: Innovations Toward the IoT, 5G, and Smart Media Era, ICTC 2015, 2015, pp. 819-821.

[12] “Open Source MANO - OSM Data Model,” 2016.

[13] B. NGMN Alliance, R. El Hattachi, and J. Erfanian, "NGMN 5G White Paper," 2015.

[14] A. Neal, "5G End-to-End Architecture Framework," Telecom Italia, 2018.

[15] A. Devlic, A. Hamidian, D. Liang, M. Eriksson, A. Consoli, and J. Lundstedt, "NESMO: Network slicing management and orchestration framework," 2017 IEEE Int. Conf. Commun. Work. ICC Work. 2017, pp. 1202- 1208, 2017.

[16] "OPNFV white paper," OPNFV PAVING W. TO OPEN SOURCE NFV, 2015.

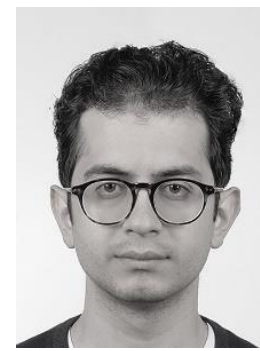

Kasra Kassai received the B.Sc. degree (Hons.) in computer science from Azad university Tehran, Iran, in 2015, the M.Sc. degree with Merit in Software Engineering from City, University of London, UK. He is currently pursuing the Ph.D. degree in Computer Science and Informatics at London South Bank University, U.K. He was a Software Developer and Test Engineer at SuperCarers Ltd., UK, between his M.Sc. and Ph.D. He has Teaching experience in Iran, Italy and UK. He has been teaching programing modules in $\mathrm{C}$ and Python to Computer Science bachelor students at London South Bank University. His primary research interests lie in the field of applying Artificial Intelligence in Software Defined Networks, Cloud Computing and Network Slicing.

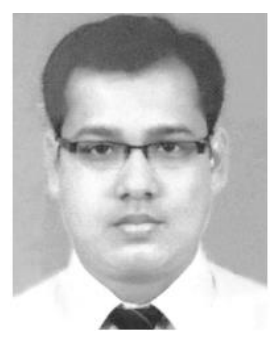

SAPTARSHI GHOSH received the B.Sc. degree (Hons.) in computer science from the University of Calcutta, India, in 2010, the M.E. degree in software engineering from Jadavpur University, India, as a GATE Scholar, in 2016, and the M.Sc. degree in smart networks from the University of the west of Scotland, U.K., as an Erasmus Mundus Scholar in 2017. He is currently pursuing the Ph.D. degree in computer science and informatics from London South Bank University, U.K., under SONNET (an MSCA-RISE project). He was a Software Developer with Webel Informatics Ltd., India, with 2 years of experience. His primary research interests lie in the field of applying machine learning in software defined networks for route optimization and self-organization in $5 \mathrm{G}$.

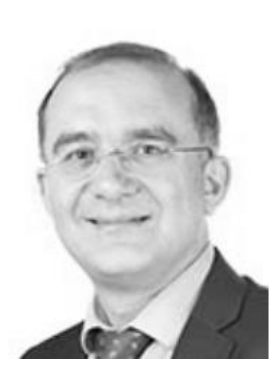

TASOS DAGIUKLAS received the Engineering Degree from the University of Patras-Greece in 1989, the M.Sc. from the University of Manchester, U.K., in 1991, and the Ph.D. degree from the University of Essex-U.K. in 1995, all in Electrical Engineering. He is a leading researcher and expert in the fields of Internet and multimedia technologies for smart cities, ambient assisted living, healthcare, and smart agriculture. He has been a principle investigator, a coinvestigator, a project and technical manager, a coordinator, and a focal person of over 20 internationally R\&D and capacity training projects with total funding of approximately č $5.0 \mathrm{~m}$ from different international organizations. He is currently the Leader of the SuITE Research Group, London South Bank University, where he also acts as the Head of the Division in Computer Science. His research interests include smart internet technologies, media optimization across heterogeneous networks, QoE, virtual reality, augmented reality and cloud infrastructure and services. 